\title{
A new generic method to extract stoichiometric and dynamic information from the exit-wave for thin sample
}

\author{
Dirk Van Dyck ${ }^{1}$, Fu-Rong Home ${ }^{2}$, Christian Kisielowski ${ }^{3}$ and Stig Helveg ${ }^{4}$ \\ ${ }^{1}$ EMAT, Department of Physics, University of Antwerp, Belgium, United States, ${ }^{2}$ Department of MSE, City \\ University of Hong Kong, United States, ${ }^{3}$ Lawrene Berkeley National Laboratory, The Molecular Foundry \\ and Joint center for Artifical Photosynthesis, University of California, Berkeley, United States, ${ }^{4}$ Center for \\ Visualizing Catalytic Processes (VISION), Department of Physics, Technical University of Denmark, DK- \\ 2800 Kgs. Lyngby, Denmark., Kongens Lyngby, Hovedstaden, Denmark
}

It has become a standard method in high-resolution transmission electron microscopy (HTREM) to record focal image series to reconstruct the exit-wave of a sample $(1,2,3,4,7,8)$. Because the exit-wave contains the most informative fingerprint of the atomic structure, it becomes utmost important to relate all this information to the atomic structure of the object in a quantitative way with the highest precision. For this purpose, a simple analytical model is needed for the exit-wave to enable model-based fitting with the experimental exit-wave. Specifically, such a model must include the dynamical electron interaction within the sample with a minimal number of parameters and must be valid up to a realistic sample thickness. Herein, we present a new model applicable to samples in a zone-axis orientation. Hereby, electrons are trapped in the atomic columns parallel to the viewing direction. The corresponding exit-wave of an atomic column reveals the projected position of the column as well as a fingerprint of the mass-thickness of the column. Based on the channeling theory (5), an analytical model is derived for the exit-wave of an atom column including also atomic vibrations and the microscopic aberrations (9). As a result, the analytical approach becomes very robust and still simple to implement. Specifically, the channeling theory $(5,6)$ describes how the electron is trapped in an atomic column parallel to the electron beam. In case of a plane wave incident beam, (5) shows that, for up to third order in the mass-thickness, the imaginary part of the exit wave is given by $\operatorname{Im}(\Psi(r, t))=\sigma \mathrm{Vp}(\mathrm{r})$ with $\mathrm{r}$ the vector in the 2D plane orthogonal to the electron beam direction, $t$ the thickness of the sample, $V p(r)$ the projected electrostatic potential of the atom column (in units $\mathrm{eV} \bullet \AA$ ),$\sigma=\pi /(\lambda \mathrm{E})$ the interaction constant and $\mathrm{E}$ the primary energy of the electron and $\lambda$ the electron wavelength. The new model extends beyond methods that only analyze the exit-wave peak values (9). Instead $\operatorname{Im}(\Psi(r, t))$ is integrated numerically over $r$ simply by adding the imaginary pixel values, which, by dividing by $\sigma$, corresponds to the projected $3 \mathrm{D}$ electrostatic atom potential and is referred to as $\mathrm{V}=\int(\mathrm{Vp}(\mathrm{r})) \mathrm{dr}$ (in units of $\mathrm{eV} \cdot \AA 3$ ). Using the Yukawa model, the 3D atom potential of an atom becomes $\mathrm{V}=\mathrm{kZe} 2 \mathrm{a} 2$. As a result, $\operatorname{Im}(\Psi(\mathrm{r}, \mathrm{t}))=\mathrm{kZe} 2 \mathrm{a} 2 \mathrm{~V}(\mathrm{r})=\alpha \mathrm{Za} 2 \mathrm{~V}(\mathrm{r})$ where the constant $\alpha=\mathrm{ke} 2$ is the same for all atoms and V(r) is a normalized atom-column-peaked function with $\int \mathrm{V}(\mathrm{r}) \mathrm{dr}$ $=1$. Furthermore, this linear approximation of $\operatorname{Im}(\Psi(\mathrm{r}, \mathrm{t}))$ is valid up to a phase change of about $0.75 \mathrm{rad}$ with an error of about $7 \%$ limiting the validity of the method to a column with a total $Z<200(5,9)$. Thus, for an image resolution sufficient to resolve neighboring columns, the linear term $\alpha \mathrm{Za} 2 \mathrm{~V}(\mathrm{r})$ provides information of the $2 \mathrm{D}$ position of the atom column and the parameter $\alpha \mathrm{Z}$ that can be fitted ("refined") as independent parameters. An important advantage of this linear interaction model is that the remaining coherent and incoherent aberrations in the exit-wave as well as the thermal vibration of the atoms can be described by convolutions in real space (6). While these effects redistribute the exit-wave over a larger area, and reduce the exit-wave peak values, the detailed blurring functions are not needed since the blurring do not affect the sum of the pixel values, which makes the method very robust. The method is employed to the analyze experimental exit-waves of a two-dimensional Co-Mo-S nanoparticle (Fig. 1a). The reconstructed exit-wave $\Psi(\mathrm{r}, \mathrm{t})$ is first normalized ("flatfielded") to the vacuum wave $\Psi \mathrm{V}(\mathrm{r})$ yielding $\Psi \mathrm{N}(\mathrm{r})=(\Psi(\mathrm{r}, \mathrm{t})-\Psi \mathrm{V}(\mathrm{r})) / \Psi \mathrm{V}(\mathrm{r})=\mathrm{i} \alpha \mathrm{Za} 2 \mathrm{~V}(\mathrm{r})$. The exit-wave is also corrected for the distance between the exit-face of a column and the plane of the reconstructed exit-wave for each atomic column. As shown in $(4,8)$, this distance can be determined with subAngstrom precision. For the resulting exit-wave, summation over all the pixel values of each exit-wave column should in principle result in one parameter $\int \operatorname{Im}(\Psi \mathrm{N}(\mathrm{r})) \mathrm{dr}=\alpha \mathrm{Za} 2$, which can be quite accurate even in case of 
a low number of imaging electrons. In order to determine Za2, the calibration factor $\alpha$ must be determined. If case of single-atom-sensitivity, the measured values $\alpha \mathrm{Za} 2$ can be plotted for all columns of the sample in one histogram and peaks at the discrete values of $\mathrm{Za} 2$ can be assigned based on e.g. pre-knowledge on the constituent atoms in the sample (9). In practice, however, the atomic columns may be blurred to an extend such that the peaks of neighboring columns overlap and the pixels cannot unambiguously be assigned specific columns. In this case, the atom column shape can be modelled by e.g. a Gaussian function with radius R. This extra parameter $\mathrm{R}$ can be determined from the pixels in the non-overlapping area and the integral under this peak can be calculated analytically. With this new approach, we analyzed the complex 3D stoichiometry and beam-stimulated dynamics of a Co-Mo-S nanocrystal at the level of single atoms (9). For quantification of mass Fig. 2(a) shows histogram of $V=\int \operatorname{Im}(\Psi N(r)) d r$ for the respective columns of $S(2$ atoms $), \operatorname{Co}(1$ atom $)$ and Mo (1 atom), and fig. 2(b) shows V/a2 as function of $\mathrm{Z}$ showing the linear relationship. Fig. 1b shows the reconstructed tomogram from analysis of Fig. 1a.

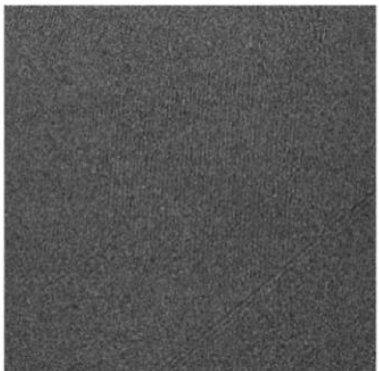

(a)

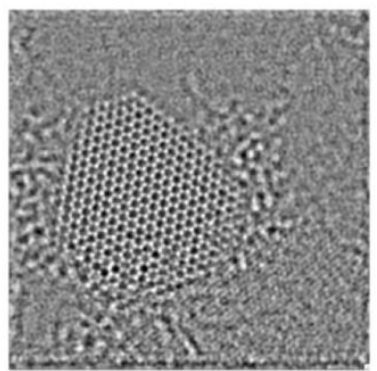

(b)

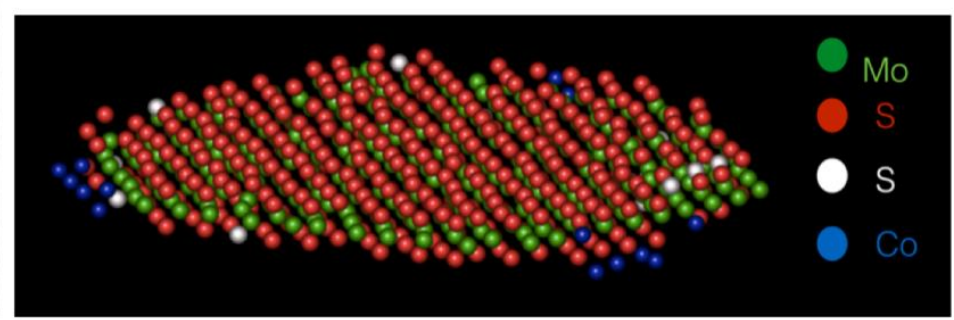

(c)

Figure 1. Fig. 1(a) A typical focal series TEM image recorded at low dose (94 e/A2.sec) (b) the phase image of reconstructed exit wave function (c) the reconstructed tomogram using the method described in this paper.

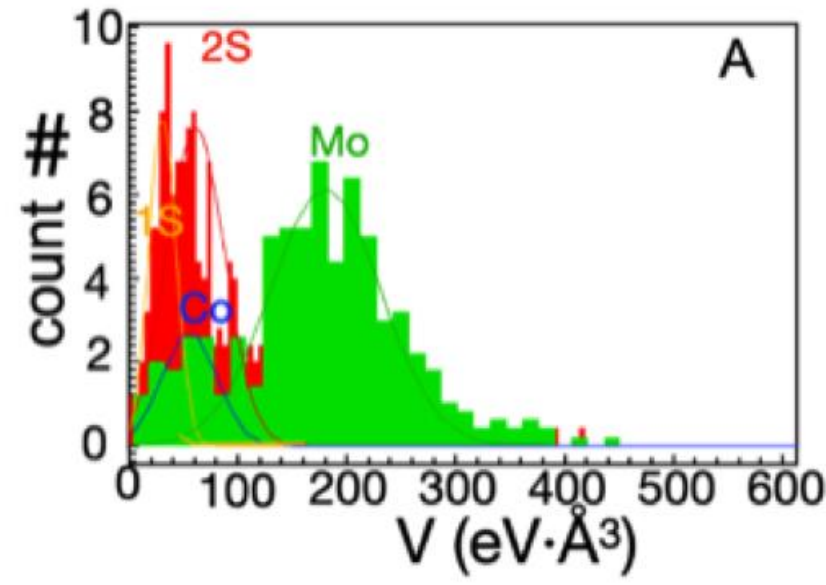

(a)

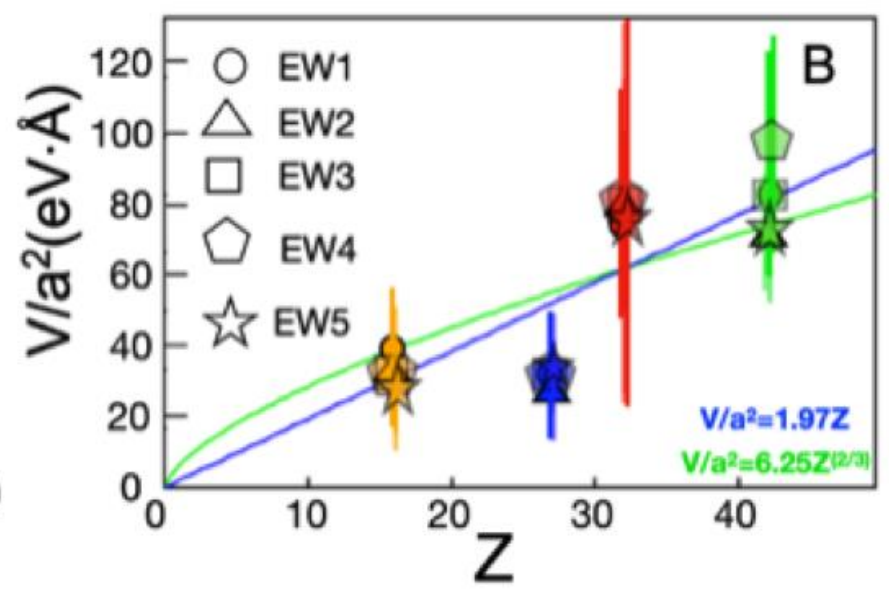

(b)

Figure 2. Figure 2 left: Histogram of $V=\int \operatorname{Im}(\Psi N(r)) d r$ for the respective columns of $S(2$ atoms $), \operatorname{Co}(1$ atom $)$ and Mo (1 atom).. Figure 2 right: V/a2 as function of $\mathrm{Z}$ showing the linear relationship. This result is consistent for 5 successive exit waves obtained with increasing total electron dose. Typical values for the atom radius a are 145, 135 and $100 \mathrm{pm}$, respectively, for Mo, Co and S. 


\section{References}

1. B. Barton, B. Jiang, C. Y. Song, P. Specht, H. Calderon, C. Kisielowski, Atomic resolution phase contrast imaging and in-line holography using variable voltage and dose rate. Microsc. Microanal. 18, 982-994 (2012). 2. W. K. Hsieh, F. R. Chen, J. J. Kai, A. I. Kirkland, Resolution extension and exit wave reconstruction in complex HREM. Ultramicroscopy. 98, 99-114 (2004).

3. F. R. Chen, C. Kisielowski, D. Van Dyck, 3D reconstruction of nanocrystalline particles from a single projection. Micron. 68, 59-65 (2015).

4. D. Van Dyck, J. R. Jinschek, F. R. Chen, "Big Bang" tomography as a new route to atomic-resolution electron tomography. Nature. 486, 243-246 (2012).

5. D. Van Dyck, M. Op De Beeck, A simple intuitive theory for electron diffraction. Ultramicroscopy. 64, 99107 (1996).

6. D. Van Dyck, in Handbook of Nanoscopy, G. Van Tendeloo, D. Van Dyck, S. J. Pennycook, Eds. (WileyVCH, 2012), pp. 45-79.

7. M. Op De Beeck, D. Van Dyck, W. Coene, Wave function reconstruction in HRTEM: The parabola method. Ultramicroscopy (1996), doi:10.1016/0304-3991(96)00058-7.

8. F. R. Chen, D. Van Dyck, C. Kisielowski, In-line three-dimensional holography of nanocrystalline objects at atomic resolution. Nat. Commun. 7, 1-11 (2016).

9. F.R Chen, D. van Dyck, C. Kisielowski, L. P. Hansen, B. Barton, S. Helveg, submitted. 